\title{
NPS and the emergent challenges for public health and psychiatry: an overview of the Portuguese situation
}

\author{
Marco Torrado $^{1 *}$, Graça Vilar ${ }^{2}$ and Domingos Duran ${ }^{3}$ \\ ${ }^{1}$ Department of Psychiatry and Medical Psychology, Faculty of Medicine, University of Lisbon, Portugal \\ ${ }^{2}$ Director of Planning and Intervention Department, SICAD - General Directorate for Intervention on Addictive Behaviors and Dependencies, Ministry of Health, Portugal \\ ${ }^{3}$ Head of Therapeutic Intervention Division, SICAD - General Directorate for Intervention on Addictive Behaviors and Dependencies, Ministry of Health, Portugal
}

\begin{abstract}
Background: The emergence of several new psychoactive substances (NPS) during last years changed dramatically the fields of addictive behaviors and the use of psychoactive substances in recreational environments, bringing new challenges for the health care systems.

Objective: Increasing knowledge on policies and the adaptations of health systems to the emergence of novel substances of abuse is critically relevant for the delivery of adequate support at different levels of care.

Methods: A review of strategic and health policy documents and surveys produced since 2012, with data related to illicit drugs and NPS collected by recognized international boards was performed. Data from Portuguese governmental entities regarding the use of NPS and indicators of associated medical interventions were also analyzed.

Results: Similar to other European countries trends, the NPS phenomenon seems to be increasing along recent years in Portugal, after a short attenuation in 2013 potentially influenced by national legal improvements. Collected data suggests that NPS users seem to adhere to community-based facilities for treatment but a relevant number of individuals are integrated in inpatient general hospitals units per year, sequentially to admissions in emergency departments due to NPS use.

Conclusion: The impact of legal mechanisms for offer reduction, prevalence of NPS use specially among adolescents and data collection in health care facilities, namely in emergency hospital admissions, need to be continuously monitored and addressed. Improvements in intervention approaches and investment in specific training programs for health professionals (e.g. GPs, psychiatrists and emergency department' physicians) are well recommended for providing adequate treatment and continuity of care.
\end{abstract}

\section{Introduction}

'New psychoactive substances' (NPS) is the chosen term adopted by the United Nations Office on Drugs and Crime (UNODC) for substances that may pose a risk to public health but are not scheduled by the Single Convention on Narcotic Drugs or even the Convention on Psychotropic Substances, dated from 1961 and 1971, respectively. But they are not necessarily a novel phenomenon. Many of these substances were synthesized in the 1970 s or even earlier, but only recently their chemistry or processes of synthesis have been modified for producing similar effects to the known illicit substances [1].

The emergence of several NPS during last years dramatically changed the fields of addictive behaviors and the use of psychoactive substances in recreational environments, bringing new challenges for public health and care system. NPS typically include substances with mainly stimulant effects, primarily hallucinogenic effects but also some with central nervous system depressant effects. Moreover many of these substances have a combination of effects, namely hallucinogenic and stimulant effects. Although the number and complexity of NPS is continuously increasing, authors have proposed some typologies to classify them. For instance, Munro \& Wilkins [2] proposed that NPS fall under five broad categories: cannabinoids; psychedelics including tryptamines and amphetamines; stimulants include substances related to cathinone, piperazine, including mephedrone and methylenedioxypyrovalerone, often sold as 'bath salts' or 'plant food' and substitute for amphetamines or ecstasy; dissociatives, including ketamine and PCP; and sedatives, including opioid substances such as morphine and fentanyl. UNODC categorized them among six main groups: synthetic cannabinoids, synthetic cathinones, ketamine, phenethylamines, piperazines and plant-based substances. A seventh group was recently proposed, the miscellaneous substances [3].

In Europe, more than 230 NPS were reported to the European Monitoring Centre for Drugs and Drug Addiction (EMCDDA) between 2005 and 2012, the diversity of which belongs to the class of synthetic cathinones [4]. In 2015 more than 450 NPS were monitored by the EMCDDA, an increasing that highlights in a very expressive way the quick evolution of this phenomenon [5].

Chemical components of most NPS are frequently modified from controlled psychoactive molecules in order to produce different psychoactive compounds, commonly sold under the label of innocuous products such as 'bath salts' and aiming to elude drug legal frameworks $[6,7]$.

Evidence has been focusing in an increasing way that many of these substances are used as 'club drugs', a short-hand term used to refer to

Correspondence to: Marco Torrado, Invited Professor, Faculty of Medicine, University of Lisbon, Medical Psychology, Av. Prof. Egas Moniz, Hospital Santa Maria 1649-028 Lisboa Lisboa, Portugal; E-mail: marcovtorrado@gmail.com

Key words: NPS, legal frameworks, levels of care, treatment, emergency rooms, training Received: June 04, 2017; Accepted: June 26, 2017; Published: June 29, 2017 
NPS typically used in dance settings, house parties and music festivals and sometimes also in a sexual context and used in chemsex practices ${ }^{\mathrm{a}}$, with methamphetamine, mephedrone, gammahydroxybutyrate/ gamma-butyrolactone $(\mathrm{GHB} / \mathrm{GHL})$ and ketamine as the substances most often reported [8,9].

Evidence also highlights that NPS are associated with a range of harms, depending on the ingested dose and biological features and/ or vulnerabilities, not only physical harms to the individuals directly caused by the substances (e.g. a wide range of cardiovascular and respiratory effects, gastrointestinal symptoms and others) but also particular neuropsychiatric conditions (e.g. psychotic and anxietyrelated symptoms), the dependence-inducing potential and finally the effects on families and society as a whole. Some studies points to an increase of deaths involving NPS recently, even though data collected still has limitations in this domain $[10,11]$.

\section{Data on NPS at the European level and the Portuguese situation}

The NPS phenomenon emerged in a more significant way in the Portuguese society almost simultaneously with the beginning of the implementation of the National Plan for the Reduction of Addictive Behaviours and Dependences 2013-2020 (National Plan) [12], implemented by the General-Directorate for Addictive Behaviours and Dependencies (SICAD). National Plan is a broad and integrated view of drug and addiction problems, involving a wide range of areas, including illicit drug use, alcohol, NPS, prescription medications, doping and gambling, accordingly with two domains: offer reduction and demand reduction.

Data from Directorate-General of Health reported 32 NPS notifications in Portugal during 2012 [13]. About 80\% were individuals aged less than 30 years old, $35 \%$ showed acute psychotic disorders and $32 \%$ showed anxiety symptoms. Among these individuals $26 \%$ registered previous episodes of NPS use.

Simultaneously, epidemiologic data collected in 2012 pointed that NPS use within 15-74 years old Portuguese population was relatively residual [14], with $0,4 \%$ reporting at least one consumption of NPS though life. Users were mainly male individuals aged between 15 and 44 years old, with prevalence up to $1 \%$, reporting NPS acquisition in smartshops in a relevant percentage ( $44 \%$ of the respondents).

Despite potential non-notified cases to health system, numbers from the previously mentioned national assessment suggested that these substances would be more used by young people, inducing psychiatric symptoms with clinical relevance and with relatively unknown longterm damages for health of young people. In some way and in parallel with international preoccupation with NPS impact in public health, these indicators supported improvements in legislation to create better responses in both legal and care systems, regarding offer and demand reduction of NPS as it shown later in this paper.

New data provided [15] by EMCDDA concerning the year of 2015 continuously point relevant indicators regarding the use of NPS, namely amphetamines and ecstasy. For example, prevalence estimates in Portuguese general population highlights a lifetime use of amphetamines in adults of $0.5 \%$, which increase in a relevant way when life-time use is considered only for 15-16 years old students (3\%). Considering the broad European scenario, recent data collected through the ESPAD survey ${ }^{\mathrm{b}}$ in 26 European countries pointed that $4 \%$

${ }^{a}$ 'Chemsex' is a term used to describe sexual practices between men that occur under the use of psychoactive substances during and/or immediately preceding sex ${ }^{\text {bESPAD Report }} 2015$ - Results from the European School Survey Project on Alcohol and Other Drugs. of the students reported lifetime experiences with NPS. The average prevalence of lifetime use of NPS was slightly higher among boys (5\%) than girls (4\%) and $3 \%$ of the students had used NPS, on average, in the last 12 months [16]. A comprehensive EU-wide survey among young people aged 15 to 24 years, the Flash Eurobarometer, reported an overall lifetime prevalence of $8 \%$ regarding NPS use [17]. Differences between these surveys (ESPAD and Flash Eurobarometer) may arise from the sampling frame (schools vs. general population) and ages (1516 years vs. 15-24 years), even though the using of the same definition of NPS.

Additionally, the use of NPS may be associated in some way to the numbers of emergency and inpatient episodes registered in last years. Supporting this assumption are the results from an European survey (leaded by Euro-DEN Plus) regarding hospital admissions in emergency departments due to psychoactive substances use. Authors highlight that presentations were predominantly established due to illicit drugs (64.3\% of substances), but in the two-year period considered $7 \%$ of substances involved in the presentations were NPS. Researchers pointed an increase of presentations involving NPS from $5.6 \%$ of reported substances between October 2013 and September 2014 , to $8.5 \%$ in year 2 (October 2014-September 2015), which is not neglectful [18].

\section{NPS and the Portuguese path traced since 2013}

\section{A. Offer reduction of NPS}

The quick expansion of the NPS phenomenon entailed, at the beginning of 2013, the approval by the Portuguese Republic Assembly of a Legal Resolution in which it was recommended to the Government the definition and endorsement of a global framework for public health protection from NPS, with technical and legal norms, and to prioritize concrete measures for dealing with the phenomenon. According with the formed consensus about the harmfulness of the NPS and its unpredictable effects for health citizens, a wide range of norms was immediately imposed against production, delivery, selling, transportation and publicity of NPS.

Laws to regulate NPS offer were produced and put into force [19]. The Decree-Law 54/2013, 17th of April, defined the legal framework for prevention and protection against publicity and commerce of NPS already acknowledged and others meanwhile identified in the market. Among other aspects, this law encompassed the potential closure of establishments selling NPS; lab analysis of NPS detected by the scientific police; procedures to be carried out by clinicians when faced with potential NPS use in hospital presentations or forensic procedures; and legal notifications of NPS holders.

Enforcement of these legal dispositions by law agencies led to a generalised elimination of smart shops activities and most of these premises were closed. Although not suppressed, selling of NPS diminished, resulting in the deletion of already existing signs of social uproar that the scaling up of this phenomenon was provoking in the Portuguese society due the perceived acute consequences for users 'health.

\section{B. Demand reduction of NPS}

\section{National framework on addictive behaviours care system}

Implementation of principles and goals of the National Plan within the framework of interventions with communities and citizens (accordingly with each type of intervention: prevention, harm and risk reduction, treatment, social integration) is put into practice considering two main axes: life-cycle phases (from unborn children to 
the elderly) and contexts where addictive behaviours and dependencies take place (Family, Community, Educational and Recreational settings, Employment, Prison).

The grid obtained by crossing these two axes enables the identification of specific groups amidst the general population that, at each life stage and context, are more vulnerable to developing addictive disorders and by so, at increased risk and in greater need of intervention. Complementarily, implementation of the major dispositions of the National Plan in the field of Treatment led to a progress in the organization of responses and interventions in this domain. An Articulation / Referral Network for Addictive Behaviours and Dependencies [20] was developed, framing not only the Specialized Centres for integrated Responses, but also the array of governmental and non-governmental institutions that deal with citizens with a broad range of addictive behaviours and dependencies. The architecture of this care network allows the involvement of all levels of health services in a stepped care model (primary care, specialized and differentiated facilities) with addictive behaviours and dependencies issues, accordingly with their degree of severity and co-occurring bio-psychosocial problems.

In what concerns services operating within the Referral / Articulation Network, their intervention is envisaged accordingly with degree of specialization of services provided, thus allowing their organization within three levels of care:

\section{- Level I: Primary care}

Provided within community-based health centres and targeted to the general population, their action is aimed at the early detection, diagnosis and assessment of the degree of severity of addictive behaviours and dependencies, and subsequently, the provision of counselling and brief interventions for low to moderate risk situations. When criteria for severe addictive behaviours are present, referral to a level II or III services is required.

\section{- Level II: Specialized care}

The main providers of outpatient treatment in Portugal are the Specialized Public Facilities, namely Specialized Treatment Teams of the Centres of Integrated Responses, Ministry of Health (ET-CRI). These specialized outpatient units, are usually the front door for the treatment system. From there, if necessary, referrals can be made to other available programs, mainly Level III inpatient units (public/ private detoxification units or therapeutic communities).

In Level II facilities clients have access to medical/psychiatric assessment, infectious diseases testing and treatment or referral, individual and group therapy, opiates substitution treatment (usually high threshold), thus ensuring a wide access to quality-controlled services encompassing several treatment modalities involving the patient and family. The services provided are free of charge and accessible to all citizens who seek treatment. To a lesser extent, NGOs and other public or private treatment services complement the network of outpatient specialized care.

\section{- Level III: Differentiated care}

Specialized medical services, namely highly differentiated public units for detoxification, psychiatry consultations and emergency departments in general hospitals, are also involved in the provision of care to addicted patients, namely in three ways: addressing patients with dual diagnosis situations, supporting patients with acute toxicity episodes due to use of certain substances and finally to provide specific interventions in the field of addictive behaviours.
Once medical stabilization is achieved by the intervention of level III services, users are frequently referred to Level II facilities (ET-CRI) but also to other general hospital departments focused on treatment of medical comorbidities (Level III).

In Portugal, with the introduction of the legal framework applicable to the consumption of narcotics and psychotropic substances (known as the decriminalization law - n. $30 / 2000$, of 29 November), people who are found by the police with a defined amount of illicit substances are referred to the 'Commissions for the Dissuasion of Drug Abuse' (CDTs) [21]. These are civil bodies operating under the Ministry of Health and provide information and advice among users, making easier the contact with care system. From CDTs many users are referred to level II facilities or others, depending on the type and severity of their problem with drugs.

\section{NPS use and responses from health care system}

\section{NPS and emergency rooms}

Considering this framework, approaches to Portuguese citizens with NPS use would involve all the three levels of care, even so available data points the emergency general hospital entries and admissions in ET-CRI facilities as the privileged front door of the care system to those NPS users.

Besides the effectiveness of the taken measures at the early stages of the NPS emergence (2013) which were able to restrain the scaling up of the phenomenon, during the following years ET-CRI continued to admit clients with NPS use, either by referral from Level III facilities, as by own initiative. Graphic 1 shows the evolution of clients admitted in those community-based units for specialized treatment, both people with NPS as primary substance use and others with NPS use within other drugs. Data is extracted from multidisciplinary registries system (SIM) used in all level II facilities at national level. All the patients inscribed in this registries' system are clinically followed in the described facilities. (Figure 1)

Analysing national data from admissions in these facilities for the last 3 years regarding the number of clients having ever used NSP, it is visible a steady decreasing trend since 2013 until 2015. Considering clients admitted that have a NSP as the main substance used, some differences in trends are perceptible, comparing with the abovementioned data on clients that have ever used NSP. In spite of the decrease registered in 2014, data from $2015(n=29)$ is similar to what was observed in 2013 ( $n=28)$, even slightly superior.

Although NSP use assumes an overall small proportion of the total treatment demands, a closer look at figures and trends for the

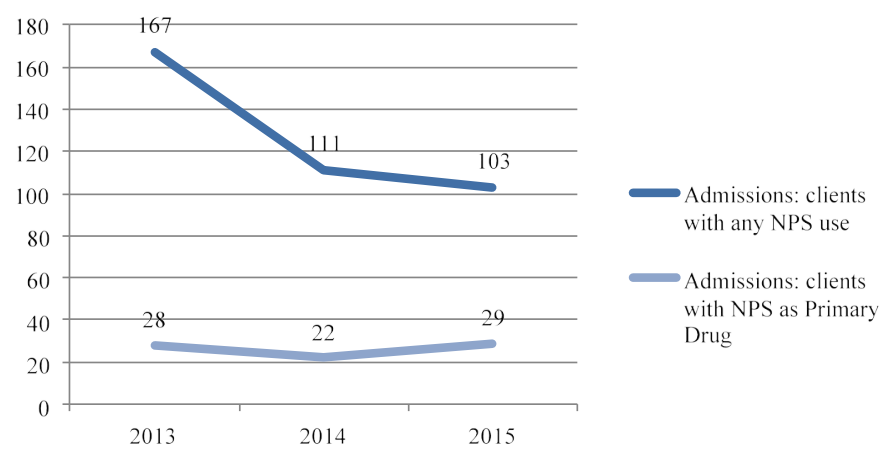

Figure 1. Number of clients admitted in level II public facilities for treating addictive behaviours with NPS between 2013 and 2015 . 
last three years enhances the idea that NSP use cannot be neglected in what concerns treatment interventions, especially due to the high costs for national health system. Data collection provided by Central Administration of Health System highlight an expressive number of admitted individuals in inpatient units after emergency episodes in general hospitals due to complications related to the use of substances like NPS and cannabis (see Graphic 2) between 2012 up to 2015. Data is based on the diagnosis codes related with the use of psychoactive substances included in ICD-9-CM (between 304.00 and 304.93; and 291.00 to 291.9 ) which are afterwards grouped and analysed as 'hospital groups of diagnoses'. Five main diagnoses groups were established to classify the need of inpatient integration. As it shown, the number of these inpatient referrals related with these novel substances are significantly higher compared to those occurred due to other substances misuse, for instance the opiates ( $\mathrm{p}=.000151)$. Moreover, it is relevant to highlight that $46 \%$ of NPS and cannabis inpatients have less that thirty years-old and about $30 \%$ have less than twenty-five, supporting the idea that people using these novel substances are younger than other users. (Figure 2)

The Graph 3 adds some support to the abovementioned considerations, in which mean ages of individuals integrated in inpatient units between 2012 and 2015 are shown. Statistical tests comparing mean ages between different users showed significant differences between NPS and cannabis users and those with consumption of opiates or cocaine $(\mathrm{p}<.05)$. (Figure 3 )

\section{Health care interventions}

The different levels of health care system need new approaches to these users, training of professionals regarding NPS and mainly diagnostic features seems to be essential to guide interventions in a proper way. Adequate training on brief interventions must be prioritized, according to the level of care.

Professionals may be allowed to contact with different brief interventions based in Motivational Interviewing (MI) principles [22], which have been used in many different situations and settings, including outpatient services and primary care services.

MI is a collaborative conversation approach for reinforcing people motivations and commitment to change. Frequently, and especially in primary care, individuals are not fully ensured or aware of their problems with drugs, particularly when its use is episodic and restricted to some recreational contexts, or they can be ambivalent

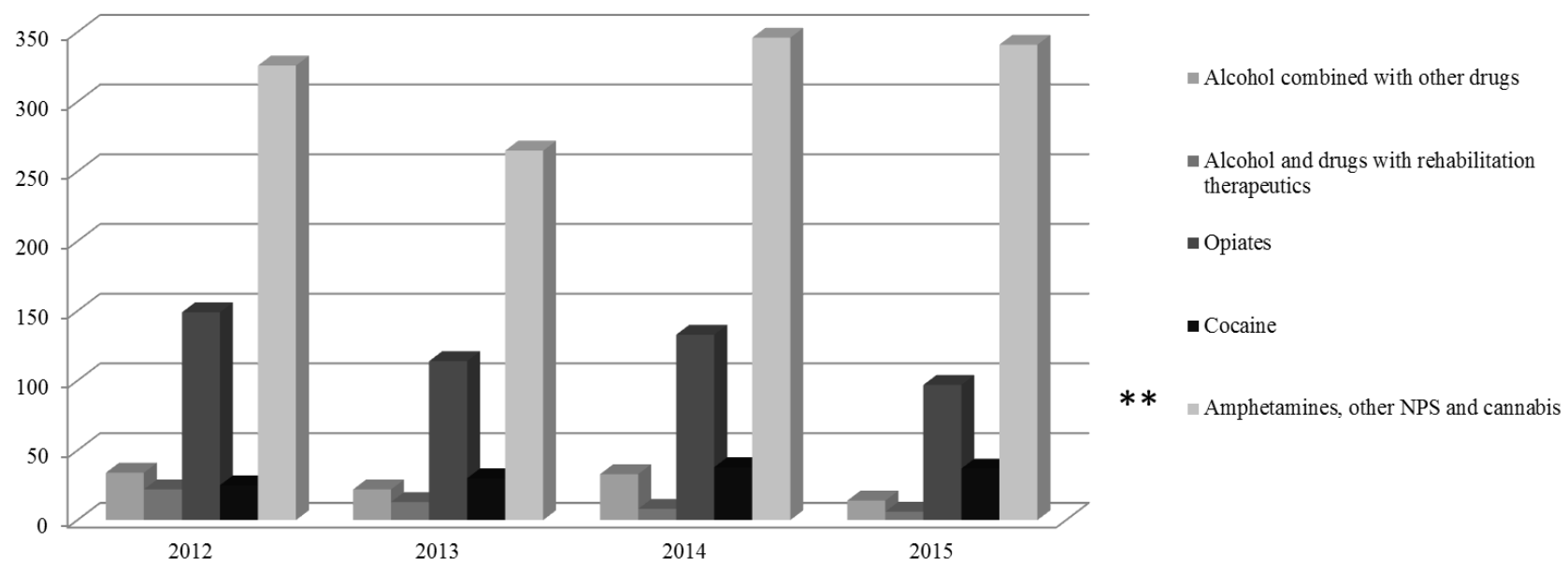

Figure 2. Number of inpatient admissions in portuguese general hospitals sequenciallyto emergency episodes related to psychoactive substances use (2012-2015)

$*$

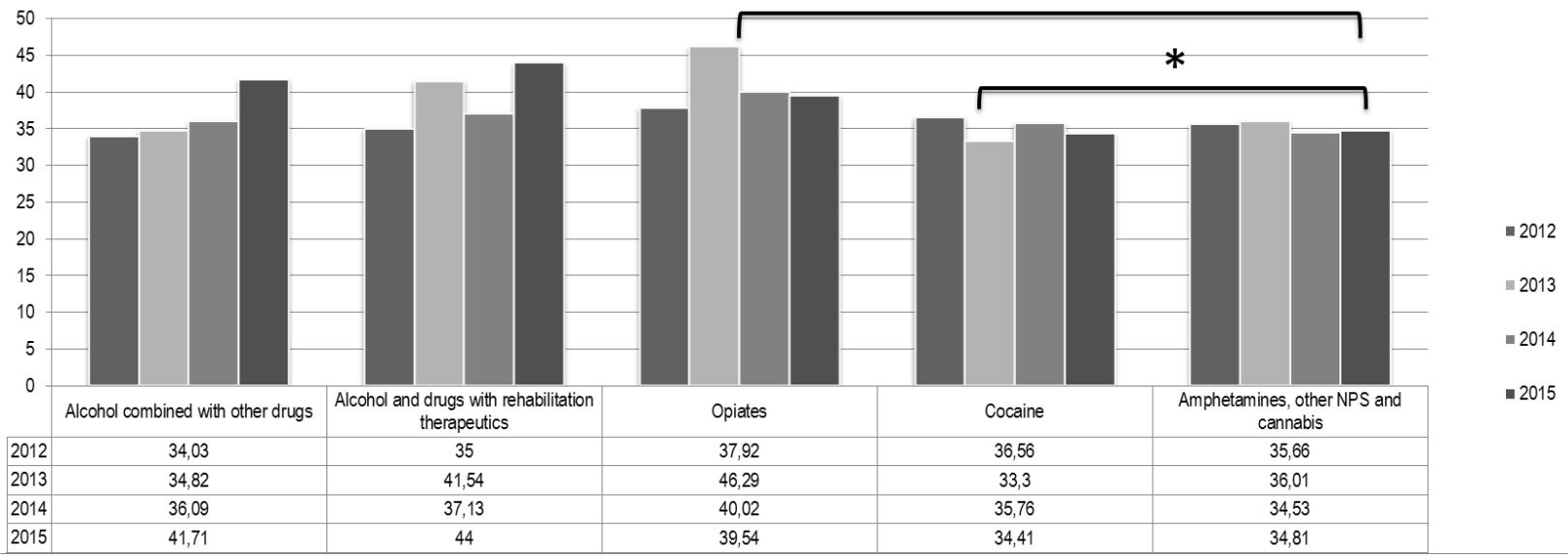

Figure 3. Mean ages of inpatients in portuguese general hospitals sequencially to emergency episodes related to psychoactive substances use (2012-2015) 
about them. MI seeks to address individuals' ambivalence about their problems with substances, as this is considered a major barrier to think about their behavioural patterns. It comprises five dimensions for intervention: expressing empathy for patients; helping patients to identify discrepancies between their goals and behaviours; avoiding arguments with patients about their behaviours and motivations; going on with patients' resistance to express certain topics and strengthening patients' self-efficacy sense. This type of intervention, empathetic and generalizable to individuals with multiple ages and problems, may be extremely relevant in a context of screening, prevention and early support, as primary care aims in the addictive behaviours domain.

Recent systematic reviews of many studies involving about large samples of participants support evidence of the benefits of these brief interventions [23], including with youth [24] as they seem to improve outcomes regarding psychoactive substances use, compared with no intervention.

Experts (e.g. Euro-DEN Plus steering group) recommend the need of introduce brief psychosocial interventions with substance users in emergency department settings [18,25], even though evidence supporting its effectiveness is still short. Since there is no acknowledged robust pharmacotherapy for treating NPS dependencies or to effectively minimize their addictive behaviours, intervention in acute toxicity episodes has privileged the use of antipsychotic medication and benzodiazepines for controlling psychiatric symptoms or supportive care for attenuate other symptoms. Many authors suggest that even necessarily recognizing the balance between providing acute medical care for patients within the emergency department and secondary approaches such as brief interventions, these types of intervention may be relevant for increasing monitoring of the NPS phenomenon, the referral to specialized appointments subsequently to the emergency or inpatient admissions and, of course, the accessibility of patients to the adequate level of care [26, 27].

\section{NPS and outreach}

In a similar way to what has been done in last decades regarding addictive behaviours and dependencies related to illicit drugs and alcohol, clinical and psychosocial support to NPS users must privilege a broad set of contexts where these substances are typically used, but also the diversity of health system settings to which people using NPS seek for care due to a diversity on health problems caused or maintained by those uses. Additionally, and since NPS use is an increasing phenomenon at different countries, adjustment in medical and clinical approaches must be thought and taken for improving accessibility and continuity of care.

The consensus around the potential injuries provided by some NPS implicates a close partnership with NGOs and associations, typically from the social sector of health, in order to complement the interventions and the achievement of main goals by the national health system in this domain. Outreach projects regarding prevention and harm reduction related to the use of psychoactive substances, namely NPS, are funded by national health system and carried out by associations and NGOs that, within formal and unformal settings, provide actions such as the following:

- Substances analyses in night-life settings to inform users about what kind of substances they are consuming, supporting their decisionmaking;

- Information about substances effects and delivery of paraphernalia for adopting less harmful forms of use;
- Information delivery and psychoeducation on sexual health and high-sexual risk behaviours typically taken when certain substances are used;

- Supportive and psychosocial care in recreational events amongst users with emergency episodes or other problems related to the NPS use.

\section{Discussion and conclusions}

In this brief report we revised relevant literature on European Health policies and frameworks regarding the use of NPS, supported in scientific knowledge that supports a wide range of related morbidities, with a special focus on psychiatric disorders. International surveys highlight the use of the substances mainly in younger people, which has been considered regarding the development of specific prevention programmes and professionals training. Special attention must be taken in what concerns to the use of psychoactive substances in young people and the potential risks of its use for health, in general, and specifically to the development of neuropsychiatric disorders at early stages.

Portuguese framework in the domain of reduction of offer and demand of NPS was also stated. Legal changes carried out since 2013 induced at some extent offer reduction and less users seeking care system. Nevertheless, the slight increasing of users seeking treatment in community-based facilities in later years and the expressive number of individuals being included in inpatient units after an emergency episode due to cannabis and NPS use are relevant indicators of the problematic patterns of use. Data included in this paper, although modest, showed a decreased number of inpatient admissions in general hospitals related to other substances excluding opiates and cocaine during 2013, which is consonant with the apparent immediate consequences of the implementation of the previous described legislation. It emphasizes that the offer reduction mechanisms provided by the regulation of smart shops had some impact, nevertheless the sales and consuming of these substances increased again in later years, perhaps more restricted to other contexts as the internet).

Additionally, available data support that users of these novel substances are younger and a part of them show medical complications entering to general hospitals' emergency departments. This fact is very concerning and may requires different public health approaches, not only addressing treatment for those people with drug misuse but also supporting new ways to help young people to resist to maladaptive patterns of using these substances, in order to achieve their full potential in society. For instance, child and adolescent mental health services should be represented in regional and national levels of decision when issues regarding young people are being addressed [28], specifically regarding new treatment approaches to young users of NPS.

Regarding this topic, literature has pointing the need of establishing motivational approaches in the multiple settings the NPS users seeking treatment, to promote a link between users and health care system which may be determinant for future interventions and the continuity of care.

Thus, regarding provision of care in NPS domain, and to achieve an integrative paradigm of medical and clinical intervention with these users towards to a more effective approach aiming demand reduction, the following actions are recommended:

1. Training of professionals from primary care and emergency departments from general hospitals, increasing their knowledge on NPS (e.g. symptoms, specific medical complications associated; therapeutics for acute toxicity and brief interventions based on 
motivational interviewing principles) but also regarding the different levels of care and the referral circuits;

2. Prioritizing regular data collection specially within emergency hospital departments and level II facilities (with well-defined indicators of accessibility and continuity of care, among others) in order to better characterize the evolution of the phenomenon;

3. Development of information resources such as booklets, leaflets and websites for GPs / primary care professionals regarding the screening of NPS use and brief interventions in primary health care;

4. Introducing thematic modules of NPS assessment, clinical management and brief psychosocial interventions in the curricula of physicians' internship, namely psychiatrists, child and adolescent psychiatrists, pediatricians and GPs, in articulation with the Professional Boards and Medical Specialty Colleges.

This paper aimed to highlight some data regarding NPS use and the impact in Portuguese health care system since 2013, after the emergence of smartshops, more notifications related to NPS use and the development of legal mechanisms that supported offer reduction in this domain. Monitoring of the legal procedures applied to these NPS acquisition need to be continuously carried out, in a close cooperation with law agencies, in order to better know what are the real impact in populations and in their patterns of use, but also to promote potential refinements of those laws according to the evolution of the phenomenon. Regarding demand reduction, brief motivational interventions are recognized as evidence-based and cost-effective tools with much potential to be applied in many different settings of care, complementing existing pharmacological interventions to mitigate acute psychiatric symptoms and provide supportive care. Effectiveness of other psychosocial interventions must be continuously studied in these populations. Training programs for health professionals seem to be well-recommended strategies for providing adequate screening, treatment and continuity of care.

\section{Acknowledgments}

The authors thank Dr. ${ }^{a}$ Claudia Medeiros Borges, from the Healthcare Financing Department of Central Administration of Health System (ACSS), Portuguese Ministry of Health, for providing data regarding inpatient admissions in general hospitals subsequent to emergency entries due to the use of psychoactive substances.

\section{Conflicts of interest}

The authors declare no conflicts of interest.

\section{Funding sources}

No grants or subsidies funded this work.

\section{References}

1. United Nations Office on Drugs and Crime (2013) The challenges of new psychoactive substances, Global SMART programme. Vienna: UNODC.

2. Munro G, Wilkins C (2014) PolicyTalk - New Psychoactive Drugs: No Easy Answer. Australian Drug Foundation.

3. Abdulrahim D, Bowden-Jones O (2015) on behalf of the NEPTUNE Expert Group. Guidance on the Management of Acute and Chronic Harms of Club Drugs and Novel Psychoactive Substances. London: Novel Psychoactive Treatment UK Network (NEPTUNE).

4. Eurosurveillance editorial team (2013) The European Monitoring Centre for Drugs and Drug Addiction publishes the European Drug Report 2013: trends and developments. Euro Surveill 18. [Crossref]
5. EMCDDA (2015) New psychoactive substances in Europe. An update from the EU Early Warning System (March 2015). Luxembourg: Publications Office of the European Union.

6. Araújo AM, Valente MJ, Carvalho M, Dias da Silva D, Gaspar H, et al. (2015) Raising awareness of new psychoactive substances: chemical analysis and in vitro toxicity screening of 'legal high' packages containing synthetic cathinones. Arch Toxicol 89: 757-771. [Crossref]

7. Baumann MH, Partilla JS, Lehner KR (2013) Psychoactive "bath salts": not so soothing. Eur J Pharmacol 698: 1-5. [Crossref]

8. Bourne A, Reid D, Hickson F, Torres Rueda S, Weatherburn P (2014) The Chemsex Study: Drug Use in Sexual Settings Among Gay and Bisexual Men in Lambeth, Southwark and Lewisham. Sigma Research. London: London School of Hygiene and Tropical Medicine.

9. Schmidt AJ, Bourne A, Weatherburn P, Reid D, Marcus U, et al. (2016) Illicit drug use among gay and bisexual men in 44 cities: Findings from the European MSM Internet Survey (EMIS). Int J Drug Policy 38: 4-12. [Crossref]

10. King LA1, Nutt DJ2; Independent Scientific Committee on Drugs (2014) Deaths from "legal highs": a problem of definitions. Lancet 383: 952. [Crossref]

11. Public Health England (2014) Turning Evidence into Practice Preventing Drug-Related Deaths.

12. SICAD (2013) Plano Nacional para a Redução dos Comportamentos Aditivos e das Dependências 2013-2020. Lisboa: SICAD.

13. Direção Geral da Saúde (2012) Notificação de casos desencadeados pelo consumo de novas substâncias psicoativas: relatório.

14. Balsa C, Vital C, Urbano C (2014) Inquérito Nacional ao Consumo de Substâncias Psicoativas na População Geral, Portugal 2012. Lisboa: SICAD.

15. Mounteney J, Griffiths P, Sedefov R, Noor A, Vicente J, et al. (2016) The drug situation in Europe: an overview of data available on illicit drugs and new psychoactive substances from European monitoring in 2015. Addiction 111: 34-48. [Crossref]

16. EMCDDA (2016) ESPAD Report 2015 Results from the European School Survey Project on Alcohol and Other Drugs. Luxembourg 2016.

17. European Commission (2016) 'Young people and drugs', Flash Eurobarometer 401.

18. EMCDDA (2016) Emergency department-based brief interventions for individuals with substance-related problems: a review of effectiveness. Luxembourg: EMCDDA Papers, Publications Office of the European Union.

19. SICAD (2013) New Psychoactive Substances: Portuguese legal framework for the prevention and protection against advertisement and commerce of new psychoactive substances. Lisboa: SICAD.

20. SICAD (2013) Decriminalisation: Portuguese legal framework applicable to the consumption of narcotics and psychotropic substances. Lisboa: SICAD

21. SICAD (2013) Rede de Referenciação / Articulação no âmbito dos Comportamentos Aditivos e das Dependências. Lisboa: SICAD.

22. Millner R, Rollnick S (2012) Motivational interviewing: Helping people change. New York: Guilford Press.

23. Smedslund G, Berg RC, Hammerstrøm KT, Steiro A, Leiknes K, et al. (2011) Motivational interviewing for substance abuse (Review), Cochrane Database of Systematic Reviews.

24. Barnett E, Sussman S, Smith C, Rohrbach LA, Spruijt-Metz D (2012) Motivational Interviewing for adolescent substance use: a review of the literature. Addict Behav 37: 1325-1334. [Crossref]

25. EMCDDA. Hospital emergency presentations and acute drug toxicity in Europe: update from the Euro-DEN Plus research group and the EMCDDA; 2016. Luxembourg: Publications Office of the European Union.

26. Bernstein E, Topp D, Shaw E, Girard C, Pressman K, et al. (2009) A preliminary report of knowledge translation: lessons from taking screening and brief intervention techniques from the research setting into regional systems of care. Acad Emerg Med 16:1225-33. [Crossref]

27. Wood DM, Heyerdahl F, Yates CB, Dines AM, Giraudon I, et al. (2014) The European Drug Emergencies Network (Euro-DEN). Clin Toxicol 52: 239-241.

28. Richardson G, Partridge I, Barrett J (2010) Child and Adolescent Mental health Services: an operational handbook. London: The Royal College of Psychiatrists 2010.

Copyright: (C2017 Torrado M. This is an open-access article distributed under the terms of the Creative Commons Attribution License, which permits unrestricted use, distribution, and reproduction in any medium, provided the original author and source are credited. 